\title{
Development of an integrated optoelectronic system for search and rescue mission control
}

\author{
M. Sajkowski, ${ }^{* 1}$ T. Stenzel, ${ }^{1}$ T. Boratyński, ${ }^{2}$ J. Bokszczanin, ${ }^{3}$ and T. Kruk ${ }^{3}$ \\ ${ }^{1}$ Department of Power Electronics, Electrical Drives and Robotics, Silesian University of Technology, \\ 2 Krzywoustego St., 44-100 Gliwice, Poland \\ ${ }^{2}$ Center for Advanced Manufacturing, Wrocław University of Science and Technology, \\ 5 Łukasiewicza St., 50-371 Wrocław, Poland \\ ${ }^{3}$ Korporacja Wschód Sp. z o.o., 28 Pożaryskiego St., 04-703 Warszawa, Poland
}

Received August 04, 2016; accepted September 10, 2016; published September 30, 2016

\begin{abstract}
In this work, the results of development of optoelectronic system designed for search and rescue maritime operation are presented. Due to integration a number of few data sources in the console of the system operator, the main focus of the paper is signal interfaces and data flow within the developed solution. The structure of the system is described as well as data flow for images and control is presented. Finally, the images showing examples of system operation are shown.
\end{abstract}

The significance of thermal imager applications in maritime service has been well known since the nineteen seventies [11]. The usefulness of such devices in surveillance, search and rescue is undisputed, but there are some special requirements for a thermal imager and for other equipment used in maritime conditions. Firstly, the optoelectronic devices designed for this kind of applications should be integrated in a single fully waterproof turret - typically three devices: a thermal imager, camera operating in visible light and laser rangefinder. The abovementioned turret should rotate in two degrees of freedom horizontally in azimuth and vertically in elevation. This equipment allows for operation during day and night and regardless of weather conditions during search and rescue missions. There are no strict definitions of an integrated optoelectronic system for search and rescue mission control, but the system should meet the criteria listed above. The described system is proposed for implementation on board of rescue vessels. There is an assumption that the system is operated by a dedicated person who is coordinating the rescue mission. The concept of the integrated system means that the operator during the mission is able to track various signals coming from different sensors: optical and navigational.

There are two main components of the described system: an optoelectronic turret and a system operator control desk. The turret can rotate continuously horizontally $(n \times$ 360 degrees). The range of operation in elevation angle is 90 degrees. The turret has been provided by Etronika

*E-mail: maciej.sajkowski@ polsl.pl company and the equipment has been manufactured according to special requirements of the maritime application. Some parts of the optoelectronic turret have been made using a rapid prototyping approach with a 3D printer operating in Selective Laser Sintering (SLS) technology. A view of the main system components is presented in Fig. 1. Thus it was possible to reduce the weight of the turret by almost $15 \%$, which was important for the selection of drives. The main parameters of a thermal camera implemented within the optoelectronic system are collected in Table 1.

\section{Table 1}

\begin{tabular}{|c|c|}
\hline Parameter & Range or value \\
\hline Resolution & $640 \times 512$ \\
\hline Spectral range & $3.7 \mu \mathrm{m}-4.8 \mu \mathrm{m}$ \\
\hline Sensitivity & NEDT $<18 \mathrm{mK}$ \\
\hline Horizontal field of view & $4-16^{\circ}$ \\
\hline
\end{tabular}

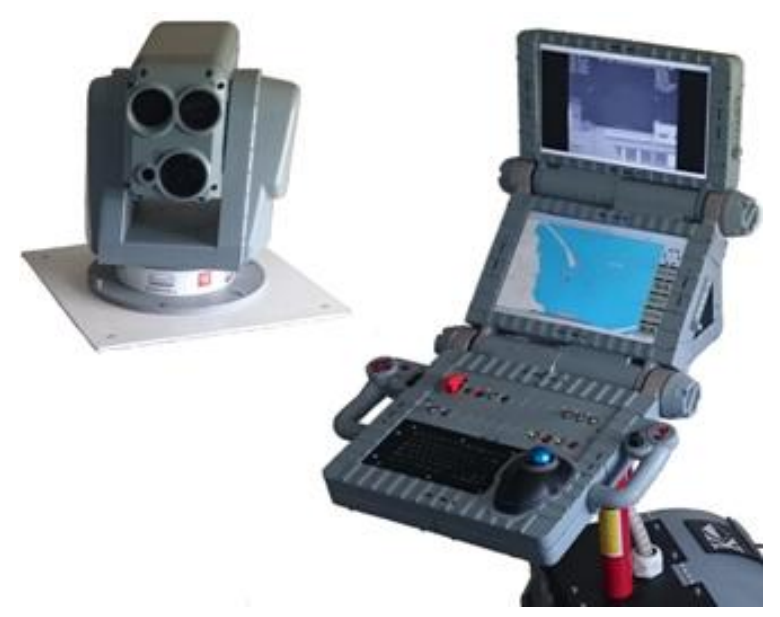

Fig. 1. Optoelectronic system for search and rescue mission control.

In the case of acquisition and processing of data 
coming from various image sensors, it is essential to achieve minimal transport delays and receive uncompressed data. This is quite similar to medical image processing, where any distortion or deformations in the image structure could lead to significant mistakes in content interpretation.

There are two main sources of image data. The first sensor is a visible light camera and the other is a thermal imager based on a focal plane array microbolometer sensor operating in MWIR. The rule of operation and detailed information about the used detector can be found, among others, in publications [2], [5], [9], [7], [10] and [8]. The optoelectronic data processing architecture as well as the control of optoelectronic turret devices are presented in Fig. 2. There are three parts of a block diagram. The upper part describes the structure of a thermal camera. The middle one presents the functional blocks of a visible light camera and the lower part shows a simplified laser rangefinder structure.

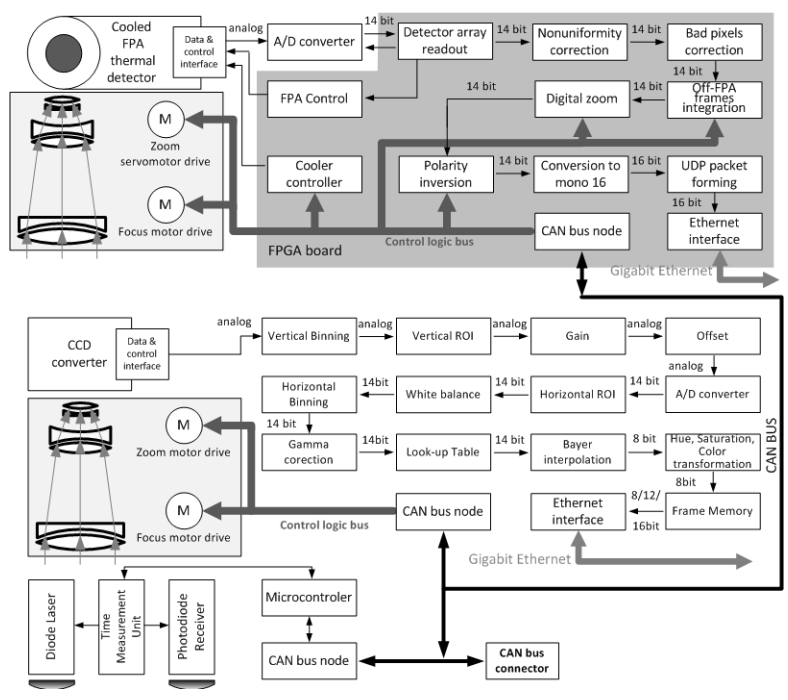

Fig. 2. Optoelectronic turret control, data acquisition and processing schematic diagram showing a thermal camera, visible light camera and laser rangefinder structures.

The block diagram of a thermal camera is representative of devices described in papers [1] and [9]. Considering the abovementioned requirements, the main interface for acquisition of optoelectronic data in the described system was GigE-vision. It allows to establish connection between the data processing unit and optoelectronic data source using typical Ethernet equipment like cables and network interface cards compatible with 1000 Mbit version of Ethernet, called Gigabit Ethernet. That approach is widely used in commercially available cameras [4] as well as in specially designed image processing surveillance [3], [6] and robotic systems [12]. The throughput of the mentioned data link is enough to receive uncompressed data from a megapixel camera. In the described solution there have been two separate network interface cards for both cameras: visible light and thermal. That approach allows to achieve optimal performance of data receiving and further processing. As it comes from Fig. 2, there are a number of operations performed on data streams generated by optoelectronic sensors. From the perspective of system development, the operations shown as sequences of blocks in the mentioned figure were implemented by manufacturers of the optoelectronic devices. In respect of real time data processing, typically, the implementation is based on FPGA circuits. There are some blocks in the upper part of Fig. 2, which are connected to a CAN bus node by a Control logic bus. The picture shows a virtual control bus, typically not available as a physical common connection between elements, but showing the operations or units, which are controlled through a CAN bus from the surveillance system operator control desk. In the case of a visible light camera the image processing operations are performed autonomously by a camera control system. It is only possible to control the values of focus and field of view (FOV) angle of a visible light camera lens as well as analogical parameters of thermal camera optics. Additionally, it is possible to set selected parameters of a thermal camera like digital zoom, image polarity and filtering thermal image by integrating a sequence of successive two, four or eight image frames. The described control architecture allows also for execution of range measurements and receiving results. Due to application of a powerful computational unit for data acquisition and visualization, it is possible to apply additional image processing operations, basing on the software developed for a Linux operating system.

In the case of various optoelectronic aerial and maritime surveillance systems there are used mainly two types of control interfaces. The first solution is based on the RS-485 or RS-422 connection and the other utilizes the CAN interface. In the described system the second approach has been applied. Two variants of optoelectronic device control were considered: one using a visual data processing computer and another one based on a separate operator desk controller. Finally, it has been decided to design the abovementioned desk control unit as a 32-bit microcontroller board equipped with digital I/O, analog inputs, and serial as well as CAN interfaces. The adopted solution allows to control the optoelectronic turret independently from the image acquisition system, so the visual data processing computer is not loaded with low level control protocols. Additionally, the status of all main system components is continuously monitored and potential errors are reported. A serial interface type RS232 is used to exchange data between the control desk for the optoelectronic turret and the visual data processing 
computer. That allows for visualizing some data received from optoelectronic devices on the operator's screen. The current horizontal and vertical angles of the turret are presented graphically as well as FOV angle. Besides, all main data including rangefinder measurements are overlaid on the image form selected camera as numbers complemented with an appropriate description. It is also possible to append the image with data coming from external devices like a gyrocompass or GPS receiver. The user interface is fully customizable, in respect of data format, position on the screen, font size as well as the format of graphical elements indicating the field of view angle and current rotation angles of the turret. At present, the user interface of the optoelectronic surveillance system is in Polish, but it is possible to switch the software to any other language.

A preliminary test of functionality of the developed system has been performed. The first stage of the test was carried out during work on the operator desk controller software. The final test has been performed on board of a research vessel S/Y Magnus Zaremba. The image from the thermal camera and that from the visible light camera operating in maritime conditions, as well as images from the thermal camera in normal and inverted polarization are shown respectively in Fig. 3 and 4.
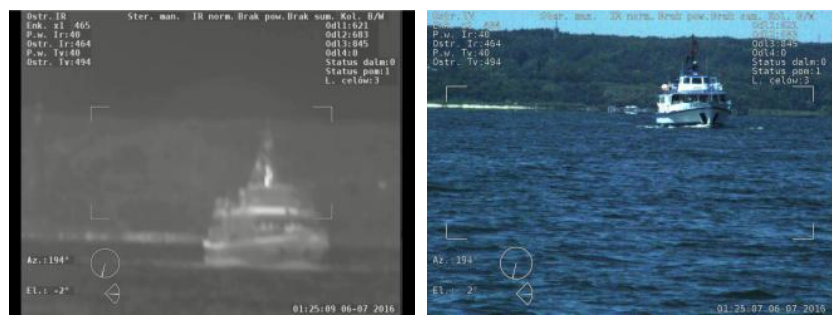

Fig. 3. Images of a vessel from thermal and visible light cameras.
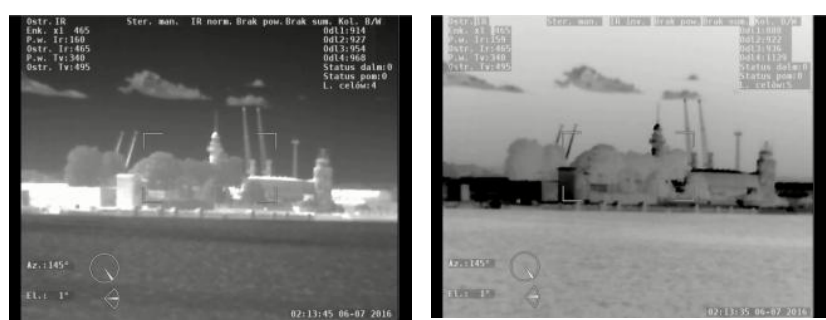

Fig. 4. Image from thermal camera taken while observing the ground infrastructure (normal and inverted polarization).

In conclusions, the paper presents the first stage of development of an optoelectronic system for maritime surveillance, especially for search and rescue applications. A specially manufactured optoelectronic turret equipped with visible light and thermal cameras, as well as a laser rangefinder have been integrated with an operator control desk and image acquisition software. Beside optoelectronic data receiving and processing, the developed system enables reception, processing and visualization of navigational data coming from a number of sensors like a GPS receiver, Automatic Identification System (AIS) receiver and Gyrocompass. The developed system may not only increase the efficiency of running search and rescue missions but also could be used as a mobile research stand for further improvement of optoelectronic data processing automation. The weight reduction of an optoelectronic turret has been achieved as well as required ergonomics of the system operator desk thanks to used lightweight and durable polyamide components produced with rapid prototyping technologies like the applied SLS. The next step of system development is the implementation and testing of a control unit for vessel motion compensation based on drives built in a turret. The aim is to achieve a more stable image. An automatic target tracking function can also be implemented, as well as position estimation based on visual detection and rangefinder measurements. Another direction of further system development is the design and construction of a testbed for optoelectronic stabilization or motion compensation of the described turret and the other optoelectronic electromechanical equipment.

The work was supported by the National Centre for Research and Development in Poland within the grant PBS1/A6/12/2012.

\section{References}

[1] G. Bieszczad, T. Sosnowski, H. Madura, M. Kastek, J. Bareła, Proc. SPIE 8012, Infrared Technology and Applications XXXVII 80120L (2011).

[2] N.K. Dhar, R. Dat, A.K. Sood in Optoelectronics - Advanced Materials and Devices, S. Pyshkin (Ed.), InTech (2013).

[3] O.W. Ibraheem, A. Irwansyah, J. Hagemeyer, M. Porrmann, U. Rueckert, 2015 International Conference on ReConFigurable Computing and FPGAs (ReConFig), 1 (2015).

[4] C.Y. Liao, C.Y. Wu, Y.S. Cheng, C.H. Kuo, K.H. Hu, K.T. Hsu, International Symposium on Computer, Consumer and Control (IS3C), 35 (2014).

[5] K.D. Nibir, D. Ravi, K.S. Ashok in Optoelectronics - Advanced Materials and Devices, S. Pyshkin (Ed.), InTech (2013); available from: http://www.intechopen.com/books/optoelectronics-advancedmaterials-and-devices/advances-in-infrared-detector-array-technology

[6] E. Norouznezhad, A. Bigdeli, A. Postula, B.C. Lovell, ACM/IEEE Second International Conference on Distributed Smart Cameras ICDSC 2008, 1 (2008).

[7] A. Rogalski, Prog. Quantum Electron. 36(2), 342 (2012).

[8] A. Rogalski, Acta Phys. Polonica A 116(3), 389 (2009).

[9] A. Rogalski, Opto-Electr. Review 21(4), 406 (2013).

[10] T. Sprafke, J.W. Beletic, Optics \& Photon. News 19, 22 (2008).

[11] J. Stachiw, D. Endicott, Oceans '79, 424 (1979).

[12] W. Yuan He, K.H. Xiao, Z. Xu, IEEE International Conference on Mechatronics and Automation, 452 (2011). 